\title{
A nonlinear observer design for an activated sludge wastewater treatment process
}

\author{
B. Boulkroune ${ }^{a}$, M. Darouach ${ }^{a}$, M. Zasadzinski ${ }^{a}$, S. Gillé ${ }^{b}$ and D. Fiorelli ${ }^{b} *$ \\ ${ }^{a}$ Centre de Recherche en Automatique de Nancy (CRAN-UMR 7039) Nancy-University - CNRS, \\ IUT de Longwy, 186 rue de Lorraine, 54400 Cosnes et Romain, France \\ ${ }^{b}$ Centre de Recherche Public Henri Tudor, LTI, Laboratoire des Technologies Industrielles, 70, \\ route de Luxembourg, L - 4009 Esch-Alzette, Luxembourg
}

\begin{abstract}
This paper treats the problem of estimating simultaneously the state and the unknown inputs of a class of nonlinear discrete-time systems. An observer design method for nonlinear Lipschitz discrete-time systems is proposed. By assuming that the linear part of this class of systems is time-varying, the state estimation problem of nonlinear system is transformed into a state estimation problem for LPV system. The stability analysis is performed using a Lyapunov function that leads to the solvability of linear matrix inequalities (LMIs). Performances of the proposed observer are shown through the application to an activated sludge process model.
\end{abstract}

Keywords : Activated sludge, wastewater treatment process, Lyapunov function, Lipschitz singular discrete-time systems, nonlinear systems, unknown inputs, LPV approach.

\section{Introduction}

The observers design of nonlinear systems has received great attention in the literature. In the continuous-time case, various state observation methods for Lipschitz systems have been proposed, see for example [1], 28], 27], [33], 26] and [24. However, few methods are presented in the discrete-time case (see [2], [20], [22], 8], [29] and [4]). In these works, the linear part is assumed to be time invariant.

In this paper, we will present an observer for state and unknown inputs estimation using the LMIs technique for a class of nonlinear Lipschitz discrete-time systems with the linear parameter-varying (LPV) approach. Our approach extends the recent results of [2] and [5].

In the last decades, the modeling of the activated sludge wastewater treatment process became an interesting area of research. The environment protection and biological wastewater treatment are essential for the life of human communities. To fulfill the requirement of the European Union as regards to environmental protection, control of the reject water quality by the wastewater treatment plants in the nature became an obligation. A Benchmark [3] has been proposed by the European program COST 624 for the evaluation of control strategies in wastewater treatment plants (http: //wWw.ensic.inpl-nancy.fr/COSTWWTP/Benchmark.htm).

\footnotetext{
${ }^{*}$ Corresponding author. Tel.: +333 8239 6222; E-mail address: Michel.Zasadzinski@iut-longwy.uhpnancy.fr
} 
Activated sludge wastewater treatment is a highly complex physical, chemical and biological process, and variations in wastewater flow rate and its composition, combined with time-varying reactions in a mixed culture of micro-organisms, make this process nonlinear and unsteady. For modeling the biological process in the activated sludge plant, several models are proposed : ASM1 (Activated Sludge Process Model No.1) [17], ASM2 [18, ASM2d [19] and ASM3 [16]. Due to the complexity of these models (for example : the ASM1 model contains 11 different components, 20 parameters and 8 processes characterized by their process rates), different versions of a reduced model for the activated sludge plant are proposed in the literature [10, [21, [31, [15], 30] and [25]. In this work, a nonlinear reduced model given by [10] is chosen for modeling the benchmark with a single Reactor Activated Sludge Process. This reduced model contains five state variables and two unknown inputs. The objective is to estimate conjointly the state and the unknown inputs. For this, we transform the nonlinear system with unknown inputs to a nonlinear descriptor system. The transformed system is composed of two parts : a linear part and a nonlinear Lipschitz part.

In practice and among the different models proposed, for modeling the activated sludge process, there are some concentrations, states or inputs, which are not measured online. To solve this problem, various methods are proposed. We can quote, for example [32, [23], 14], [11], 34] and lately [6] and references therein. Reference [11] gives an excellent overview of available results on the state and parameter estimation approaches for chemical and biochemical processes. In this paper, we will propose an observer which takes into account the Lipschitz property of the nonlinear part.

This paper is structured as follows. In the second section, an observer design for a class of nonlinear discrete-time systems using LPV approach is introduced. In the third section, the reduced model of the activated sludge process is presented and the effectiveness of the proposed observer is shown via the reduced order model of the activated sludge process in the fourth section. Finally, the fifth section concludes the paper.

Notations : The following notations will be used throughout this work :

- $\|$.$\| is the usual Euclidean norm;$

- $(*)$ is used for blocks induced by symmetry;

- $A^{T}$ represents the transposed matrix of $A$;

- $I_{s}$ represents the identity matrix of dimension $s$.

\section{Nonlinear observer design for Lipschitz discrete-time sys- tems}

In this section, we introduce our main contribution. A nonlinear observer for Lipschitz discrete-time system using LPV approach is derived. The analysis of its stability is performed by the use of the Lyapunov stability.

Consider a nonlinear discrete-time descriptor system described by

$$
\begin{aligned}
E x_{k+1} & =A\left(\varrho_{k}\right) x_{k}+f\left(x_{k}, u_{k}\right) \\
y_{k} & =H x_{k}
\end{aligned}
$$

where $A\left(\varrho_{k}\right)=A_{0}+\varrho_{k} A_{1}$ and $E \in \Re^{q \times n}, A_{0} \in \Re^{q \times n}, A_{1} \in \Re^{q \times n}, H \in \Re^{p \times n}$ are known constant matrices with $q \leqslant n$, when $q=n, E$ is singular. The variable $\varrho_{k}$ is the scheduling variable measurable online. The vector $x_{k} \in \Re^{n}$ represents the state vector, $u_{k} \in \Re^{r}$ is the input vector, and $y_{k} \in \Re^{p}$ denotes the measured output system. The function $f$ is a nonlinear function. 
Our objective is to design an asymptotic observer to estimate the state $x_{k}$. The following assumptions are made throughout the paper.

Assumption 1. The nonlinearity $f\left(x_{k}, u_{k}\right)$ is assumed to be Lipschitz in $x$ with a known Lischitz constant $\gamma$, i.e.

$$
\left\|f\left(x_{1, k}, u_{k}\right)-f\left(x_{2, k}, u_{k}\right)\right\| \leqslant \gamma\left\|x_{1, k}-x_{2, k}\right\|
$$

for all $x_{1}, x_{2} \in \Re^{n}$ and $\gamma>0$ is independent of $u_{k}$.

Assumption 2. In this paper, we assume that the following condition holds [12], [13]

$$
\operatorname{rank}\left[\begin{array}{c}
E \\
H
\end{array}\right]=n
$$

The dynamical matrix $A\left(\varrho_{k}\right)$ depends on a time-varying parameter $\varrho_{k}$. We assume that the parameter $\varrho_{k}$ ranges between known extremal values $\varrho_{k} \in[\varrho, \bar{\varrho}]$. Let us introduce the following notations

$$
\underline{A}=A(\underline{\varrho}) \quad \text { and } \quad \bar{A}=A(\bar{\varrho}) .
$$

Remark 1. The result presented in this paper can be extended easily to the case where $A\left(\varrho_{k}\right)=A_{0}+\sum_{i=1}^{j} \varrho_{i, k} A_{i, k}$.

Consider the following state observer for system (1)

$$
\begin{aligned}
z_{k+1} & =N\left(\varrho_{k}\right) z_{k}+L\left(\varrho_{k}\right) y_{k}+g\left(z_{k}, u_{k}\right) \\
\hat{x}_{k} & =z_{k}+Q y_{k}
\end{aligned}
$$

where $\hat{x}_{k}$ is the state estimation vector of $x_{k}$. Matrices $N, L, Q$ and the nonlinear vector field $g\left(z_{k}, u_{k}\right)$ must be determined such that $\hat{x}_{k}$ converges asymptotically to $x_{k}$. From [5], the parameter-varying gain matrices $N$ and $L$ can be chosen affine in $\varrho_{k}$ and are obtained by an interpolation of the gains $N_{i}$ and $L_{i}$, respectively

$$
\begin{gathered}
N=N_{0}+\varrho_{k} N_{1}, \\
L=L_{0}+\varrho_{k} L_{1} .
\end{gathered}
$$

Under assumption 2, there exist two matrices $T$ and $Q$ such as

$$
T E+Q H=I_{n}
$$

The estimation error is

$$
e_{k}=\hat{x}_{k}-x_{k},
$$

then by substituting $(5 \mathrm{~b})$ and $(1 \mathrm{~b})$ into $(8)$ we obtain

$$
e_{k}=z_{k}+\left(Q H-I_{n}\right) x_{k}
$$

and by using (7), relation (9) becomes

$$
e_{k}=z_{k}-T E x_{k}
$$


then, the dynamics of the estimation error is given by

$$
e_{k+1}=z_{k+1}-T E x_{k+1} .
$$

From (1a) and (5a), we obtain

$$
e_{k+1}=N\left(\varrho_{k}\right) e_{k}+\left(N\left(\varrho_{k}\right)+F\left(\varrho_{k}\right) H-T A\left(\varrho_{k}\right)\right) x_{k}+g\left(z_{k}, u_{k}\right)-T f\left(x_{k}, u_{k}\right)
$$

with

$$
F\left(\varrho_{k}\right)=L\left(\varrho_{k}\right)-N\left(\varrho_{k}\right) Q .
$$

By defining $N\left(\varrho_{k}\right)$ and $g\left(z_{k}, u_{k}\right)$ as follows

$$
N\left(\varrho_{k}\right)=T A\left(\varrho_{k}\right)-F\left(\varrho_{k}\right) H
$$

and

$$
\begin{aligned}
g\left(z_{k}, u_{k}\right) & =T f\left(\hat{x}_{k}, u_{k}\right) \\
& =T f\left(z_{k}+Q y_{k}, u_{k}\right),
\end{aligned}
$$

the error dynamics $(12)$ becomes

$$
e_{k+1}=\left(T A\left(\varrho_{k}\right)-F\left(\varrho_{k}\right) H\right) e_{k}+T \triangle f_{k}
$$

where

$$
\triangle f_{k}=f\left(\hat{x}_{k}, u_{k}\right)-f\left(x_{k}, u_{k}\right) .
$$

From (6) and (13), we can deduce that the matrix $F$ is affine in $\varrho_{k}$ and is given by the following interpolation

$$
F\left(\varrho_{k}\right)=F_{0}+\varrho_{k} F_{1}
$$

where $F_{0}$ and $F_{1}$ are constant matrices to be determined such that the estimation error converges asymptotically towards zero.

The problem is reduced to find the gain matrix $F$. Before giving the method of the design of this gain, we can summarize the procedure as follows : since $E$ and $H$ are known, from (7) we can deduce matrices $T$ and $Q$. Then after calculating matrices $F_{0}$ and $F_{1}$, we can deduce the matrix $F$. Matrix $N$ and be obtained from $(14)$ and then we can deduce $L$ from $(13)$.

The following theorem gives sufficient conditions for the existence of matrix $F$.

Theorem 1. The estimation error (8) converges asymptotically towards zero if there exist a scalar $\tau>0$ and matrices $P=P^{T}>0$ and $R$ of appropriate dimensions such that the following linear matrix inequalities (LMIs) are satisfied.

$$
\left[\begin{array}{ccc}
-P+\tau \gamma^{2} I_{n} & \underline{A}^{T} T^{T} P T-H^{T} R_{0} T-\varrho H^{T} R_{1} T & \underline{A}^{T} T^{T} P-H^{T} R_{0}-\underline{\varrho} H^{T} R_{1} \\
* & T^{T} P T-\tau I_{n} & 0
\end{array}\right]<0
$$

and

$$
\left[\begin{array}{ccc}
-P+\tau \gamma^{2} I_{n} & \bar{A}^{T} T^{T} P T-H^{T} R_{0} T-\bar{\varrho} H^{T} R_{1} T & \bar{A}^{T} T^{T} P-H^{T} R_{0}-\bar{\varrho} H^{T} R_{1} \\
* & T^{T} P T-\tau I_{n} & 0
\end{array}\right]<0 .
$$

In this case, the gain matrices $F_{0}$ and $F_{1}$ are given by $F_{0}=P^{-1} R_{0}^{T}$ and $F_{1}=P^{-1} R_{1}^{T}$. 
Proof. Consider the following quadratic Lyapunov function

$$
V(k)=e_{k}^{T} P e_{k}
$$

where $P=P^{T}>0$. The difference of $V(k)<0$ along the solutions of 116$)$ is given by

$$
\begin{aligned}
\triangle V(k) & =V(k+1)-V(k) \\
& =e_{k}^{T}\left(T A\left(\varrho_{k}\right)-F\left(\varrho_{k}\right) H\right)^{T} P\left(T A\left(\varrho_{k}\right)-F\left(\varrho_{k}\right) H\right) e_{k} \\
& +2 e_{k}^{T}\left(T A\left(\varrho_{k}\right)-F\left(\varrho_{k}\right) H\right)^{T} P T \triangle f_{k}+\triangle f_{k}^{T} T^{T} P T \triangle f_{k}-e_{k}^{T} P e_{k}
\end{aligned}
$$

which is equivalent to

$$
\triangle V(k)=\zeta_{k}^{T}\left[\begin{array}{cc}
N\left(\varrho_{k}\right)^{T} P N\left(\varrho_{k}\right)-P & N\left(\varrho_{k}\right)^{T} P T \\
* & T^{T} P T
\end{array}\right] \zeta_{k}
$$

where

$$
\zeta_{k}=\left[\begin{array}{ll}
e_{k}^{T} & \triangle f_{k}^{T}
\end{array}\right]^{T}
$$

From (2) and (16), we have

$$
\Gamma_{k}=\gamma^{2} e_{k}^{T} e_{k}-\triangle f_{k}^{T} \triangle f_{k} \geqslant 0 .
$$

Consequently, $\forall \tau>0$, we have

$$
\begin{aligned}
\triangle V(k) & \leqslant \triangle V(k)+\tau \Gamma_{k} \\
& \leqslant \zeta_{k}^{T}\left[\begin{array}{cc}
N\left(\varrho_{k}\right)^{T} P N\left(\varrho_{k}\right)-P+\tau \gamma^{2} I_{n} & N\left(\varrho_{k}\right)^{T} P T \\
* & T^{T} P T-\tau I_{n}
\end{array}\right] \zeta_{k}
\end{aligned}
$$

for all $\varrho_{k} \in[\underline{\varrho}, \bar{\varrho}]$. The difference $\Delta V(k)<0$ if

$$
\left[\begin{array}{cc}
N\left(\varrho_{k}\right)^{T} P N\left(\varrho_{k}\right)-P+\tau \gamma^{2} I_{n} & N\left(\varrho_{k}\right)^{T} P T \\
* & T^{T} P T-\tau I_{n}
\end{array}\right]<0
$$

for all $\varrho_{k} \in[\varrho, \bar{\varrho}]$, or equivalently, by using the Schur complement [7], to

$$
\left[\begin{array}{ccc}
-P+\tau \gamma^{2} I_{n} & N\left(\varrho_{k}\right)^{T} P T & N\left(\varrho_{k}\right)^{T} P \\
* & T^{T} P T-\tau I_{n} & 0 \\
* & * & -P
\end{array}\right]<0 .
$$

Then, as $(29)$ is affine in the parameter $\varrho_{k}$, the inequality $(29)$ is satisfied for all possible $\varrho_{k} \in[\varrho, \bar{\varrho}]$ if it is satisfied on the vertices of $[\underline{\varrho}, \bar{\varrho}]$. By putting $P F_{0}=R_{0}^{T}$ and $P F_{1}=R_{1}^{T}$, we deduce that the inequality $(29)$ is equivalent to $(19)-(20)$. This completes the proof.

The observer design for nonlinear systems presented in this paper is different from those presented in the literature for the discrete-time case. Reference [2] proposed an observer design for a class of standard Lipschitz nonlinear discrete-time systems by assuming that the matrix $A$ of the linear part is constant. The results of our paper are more general than the work presented in [2], [13] and [4]. Our results consider the general descriptor systems and their application to state and unknown inputs estimation. 


\section{The activated sludge process}

A WasteWater Treatment Plant (WWTP) usually consists of a set of activated sludge tanks, combined with a sedimentation tank, with a range of electron acceptor conditions occurring in the tanks. Depending on the concentrations of dissolved oxygen $\left(S_{O}\right)$ and nitrate present in the tanks, aerobic (oxygen present), anoxic (nitrate present, no oxygen) or anaerobic (no oxygen, no nitrate) tanks can be distinguished. Figure 1 shows a typical activated sludge WWTP layout, without considering the different pretreatment steps that normally precede the activated sludge tanks. The term WWTP model is used to indicate the ensemble of activated sludge model, hydraulic model, oxygen transfer model and sedimentation tank model needed to describe an actual WWTP. The term activated sludge model is used in this paper to indicate a set of differential equations that represent the biological (and chemical) reactions taking place in one activated sludge tank.

The "simulation benchmark" plant design is comprised of five reactors in series with a 10-layer secondary settling tank. Figure 1 shows a schematic representation of the layout.

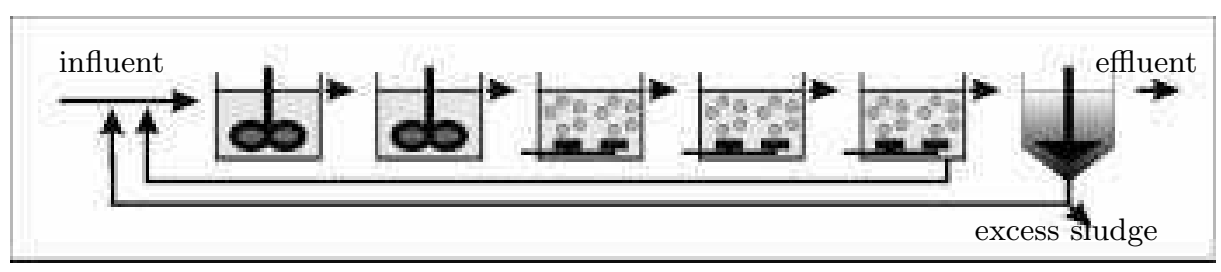

Figure 1: Schematic representation of the "simulation benchmark" configuration showing tanks 1 and 2 mixed and unaerated, and tanks 3, 4 and 5 aerated.

In this work, we consider only a part of the COST Benchmark. We have chosen the third tank with a settler as shown in figure 1. We assume, because of absence of measurement in the settler tank, that this one is perfect, i.e. no sludge leaves by the overflow the settler tank. The COST Benchmark has been proposed by the European program COST 624 for the evaluation of control strategies in wastewater treatment plants [3. The Benchmark is based on the most common wastewater treatment plant : a continuous flow activated sludge plant, performing nitrification and pre-nitrification. In this work and for simplicity, we will take only the case of one aerated tank with a settler. The volume of the tank is $1333 \mathrm{~m}^{3}$. The objective of this study is to use the data obtained from the simulations of the results in the Bleesbrück wastewater plant (in Luxembourg). Note that the measured concentrations of this station are the dissolved oxygen $\left(S_{O}\right)$, concentration that is routinely measured in activated sludge wastewater treatment plant, both nitrate $\left(S_{N O}\right)$ and ammonia $\left(S_{N H}\right)$ concentrations can be also measured online. Using the Software SIMBA and ASM1 model, the data are generated by the team of modeling and simulation of the LTI-CRP Henri Tudor in Luxembourg (see http://www.ensic.inpl-nancy.fr/COSTWWTP/Benchmark.htm). The comparison between the data generated by ASM1 model and the data from simulation of the reduced model presented in this paper is not evoked here. In this paper, only the dry weather files are used.

In this section a reduced model of the ASM1 model will be presented briefly. This model is based on the reduced nonlinear model given by [10].

- Simplification of model dynamic. Theory of the singular perturbations makes possible to consider that $X_{I}, X_{B H}$ and $X_{B A}$ have slow dynamic. Thus these variables are considered constant over a few days. Eliminating these 3 state variables, along 
with the concentrations of soluble inert organic compounds $\left(S_{I}\right)$, resulted in a 7 dimensional dynamic model.

- Simplification of the organic compounds. The measurement of the chemical oxygen demand (COD) does not make possible to distinguish between the soluble part $\left(S_{S}\right)$ and the particulate part $\left(X_{S}\right)$ [30]. A single organic compound (denoted as $\left.X_{D C O}\right)$ is formed by adding soluble and particulate organic compound concentrations.

- Simplification of the nitrogenized compounds. The mathematical expression that describes the organic nitrogen hydrolysis process is simplified so that the dynamics with respect to soluble and particulate organic nitrogen are independent. We have chosen to use only the soluble organic nitrogen $S_{N D}$.

Unlike in [9], the concentration of $S_{N O}^{i n}$ will be taken into account in the proposed model here. The reduced nonlinear model is composed of five variables : biodegradable substrate $X_{D C O}$, nitrate concentration $S_{N O}$, ammonia concentration $S_{N H}$, soluble biodegradable organic nitrogen concentration $S_{N D}$ and dissolved oxygen concentration $S_{O}$. The reduced nonlinear model is given by the following set of equations

$$
\begin{aligned}
\dot{X}_{D C O} & =D^{i n}\left(X_{D C O}^{i n}-\frac{K_{S}}{K_{D C O}} X_{D C O}\right)-\frac{1}{Y_{H}}\left(\rho_{1}+\rho_{2}\right)+\theta_{2} \\
\dot{S}_{N O} & =D^{i n}\left(S_{N O}^{i n}-S_{N O}\right)-\frac{1-Y_{H}}{2.86 Y_{H}} \rho_{2}+\frac{1}{Y_{A}} \rho_{3} \\
\dot{S}_{N H} & =D^{i n}\left(S_{N H}^{i n}-S_{N H}\right)-i_{N B M}\left(\rho_{1}+\rho_{2}\right)-\frac{1}{Y_{A}} \rho_{3}+\rho_{6} \\
\dot{S}_{N D} & =D^{i n}\left(S_{N D}^{i n}-S_{N D}\right)-\rho_{6}+\rho_{8} \\
\dot{S}_{O} & =-D^{i n} S_{O}-\frac{1-Y_{H}}{Y_{H}} \rho_{1}-4.57 \frac{1}{Y_{A}} \rho_{3}+k_{L^{a}}\left(S_{O}^{s a t}-S_{O}\right)
\end{aligned}
$$

with

$$
\begin{aligned}
\rho_{1} & =\theta_{1} \frac{X_{D C O}}{X_{D C O}+K_{D C O}} \frac{S_{O}}{S_{O}+K_{O, H}}, \\
\rho_{2} & =\theta_{1} \eta_{N O, g} \frac{X_{D C O}}{X_{D C O}+K_{D C O}} \frac{K_{O, H}}{K_{O, H}+S_{O}} \frac{S_{N O}}{S_{N O}+K_{N O}} \\
\theta_{2} & =\left(1-f r_{X I}\right)\left(\rho_{4}+\rho_{5}\right), \quad \frac{1}{Y_{A}} \rho_{3}=\theta_{3} \frac{S_{N H}}{S_{N H}+K_{N H, A}} \frac{S_{O}}{S_{O}+K_{O, A}} \\
\rho_{4} & =b_{H} X_{B, H}, \quad \rho_{5}=b_{A} X_{B, A}, \quad \rho_{6}=\theta_{4} S_{N D} \\
\rho_{8} & =\theta_{5} \frac{X_{D C O}}{X_{D C O}+K_{N D}}\left(\frac{S_{O}}{S_{O}+K_{O, H}}+\eta_{N O, h} \frac{K_{O, H}}{K_{O, H}+S_{O}} \frac{S_{N O}}{S_{N O}+K_{N O}}\right)
\end{aligned}
$$

and

$$
\begin{aligned}
\theta_{1} & =\mu_{H} X_{B, H}, \quad \theta_{3}=\frac{\mu_{A}}{Y_{A}} X_{B, A}, \quad \theta_{4}=\kappa_{a} X_{B, H}, \quad \theta_{5}=\kappa_{h} \frac{X_{N D}}{X_{S}} X_{B, H} \\
K_{D C O} & =K_{S} \frac{X_{D C O}}{S_{S}}=\frac{K_{S}}{f_{S S}}, \quad K_{N D}=K_{X} \frac{X_{D C O}}{X_{S}} X_{B, H}, \quad D^{i n}=\frac{Q_{i n}}{V_{O}} .
\end{aligned}
$$

The various values of the kinetic and stoichiometric parameters of the ASM1 model are presented in table 1. The values of the specific parameters $\theta_{i}, K_{D C O}$ and $K_{N D}$ which 
are calculated in function of the variables and parameters of the ASM1 model and other parameters are given in table 2 .

Let us take the sample time $T_{s}$, then the discretized model can be written as follows

$$
\begin{aligned}
\xi_{k+1} & =J \xi_{k}+\phi\left(\xi_{k}, u_{k}\right)+B d_{k} \\
y_{k} & =C \xi_{k}
\end{aligned}
$$

where the state, input, output and unknown input vectors are defined as

$$
\begin{aligned}
& \xi=\left[\begin{array}{lllll}
X_{D C O} & S_{N O} & S_{N H} & S_{N D} & S_{O}
\end{array}\right]^{T} \\
& u=\left[\begin{array}{ll}
\theta_{2} & k_{L^{a}}
\end{array}\right]^{T} \\
& y=\left[\begin{array}{lll}
S_{N O} & S_{N H} & S_{O}
\end{array}\right]^{T} \\
& d=\left[\begin{array}{ll}
T_{s} D^{i n} S_{N O}^{i n} & T_{s} D^{i n} S_{N H}^{i n}
\end{array}\right]^{T}
\end{aligned}
$$

and

$$
\begin{aligned}
& J=\left[\begin{array}{ccccc}
1-T_{s} D^{i n} \frac{K_{S}}{K_{D C O}} & 0 & 0 & 0 & 0 \\
0 & 1-T_{s} D^{i n} & 0 & 0 & 0 \\
0 & 0 & 1-T_{s} D^{i n} & T_{s} \theta_{4} & 0 \\
0 & 0 & 0 & 1-T_{s}\left(D^{i n}-\theta_{4}\right) & 0 \\
0 & 0 & 0 & 0 & 1-T_{s} D^{i n}
\end{array}\right] \\
& B=\left[\begin{array}{ll}
0 & 0 \\
1 & 0 \\
0 & 1 \\
0 & 0 \\
0 & 0
\end{array}\right], \quad C=\left[\begin{array}{lllll}
0 & 1 & 0 & 0 & 0 \\
0 & 0 & 1 & 0 & 0 \\
0 & 0 & 0 & 0 & 1
\end{array}\right] \\
& \phi\left(\xi_{k}, u_{k}\right)=\left[\begin{array}{c}
-T_{s} \frac{1}{Y_{H}}\left(\rho_{1}+\rho_{2}\right)+T_{s} u_{1, k}+T_{s} D^{i n} X_{D C O}^{i n} \\
-T_{s} \frac{1-Y_{H}}{2.86} \rho_{2}+T_{s} \frac{1}{Y_{A}} \rho_{3} \\
-T_{s} i_{N B M}\left(\rho_{1}+\rho_{2}\right)-T_{s} \frac{1}{Y_{A}} \rho_{3} \\
T_{s} \rho_{8}+T s D^{i n} S_{N D}^{i n} \\
-T_{s} \frac{1-Y_{H}}{Y_{H}} \rho_{1}-4.57 T_{s} \frac{1}{Y_{A}} \rho_{3}+T_{s} u_{2, k}\left(S_{O}^{s a t}-\xi_{5, k}\right)
\end{array}\right] .
\end{aligned}
$$

Now, let us introduce the following notations

$$
J(\varrho)=J_{0}+\varrho J_{1}
$$

with

$$
\varrho=D^{i n}, \quad J_{0}=\left[\begin{array}{ccccc}
1 & 0 & 0 & 0 & 0 \\
0 & 1 & 0 & 0 & 0 \\
0 & 0 & 1 & T_{s} \theta_{4} & 0 \\
0 & 0 & 0 & 1-T_{s} \theta_{4} & 0 \\
0 & 0 & 0 & 0 & 1
\end{array}\right], \quad J_{1}=\left[\begin{array}{ccccc}
-T_{s} \frac{K_{S}}{K_{D C O}} & 0 & 0 & 0 & 0 \\
0 & -T_{s} & 0 & 0 & 0 \\
0 & 0 & -T_{s} & 0 & 0 \\
0 & 0 & 0 & -T_{s} & 0 \\
0 & 0 & 0 & 0 & -T_{s}
\end{array}\right] \text {, }
$$

then, the system $(35)$ can be written as follows

$$
\begin{aligned}
\xi_{k+1} & =J(\varrho) \xi_{k}+\phi\left(\xi_{k}, u_{k}\right)+B d_{k} \\
y_{k} & =C \xi_{k}
\end{aligned}
$$

where the matrices $J(\varrho), B$ and $C$ are given respectively by (43) and (41). 
Remark 2. In the Bleesbrück station (in Luxembourg), the concentrations $X_{D C O}^{i n}$ and $S_{N D}^{i n}$ are not measured online. If we take these concentrations as unknown inputs, the system becomes unobservable. To avoid this problem, we will take the daily mean values for these concentrations, this approximation is often used in the practice.

Remark 3. In this paper, we are interested in the wastewater treatment station with only a period aerobic. We assume that this station does not have a period of significant anoxia $\left(S_{O 2}=0, k_{L^{a}}=0\right)$.

\section{Simulation results}

In this section, simulation results are provided to show the performance of the proposed approach. Let us define the augmented state $x_{k}=\left[\begin{array}{ll}\xi_{k}^{T} & d_{k-1}^{T}\end{array}\right]^{T} \in \Re^{n+m}$, system (45) can be written as follows

$$
\begin{aligned}
E x_{k+1} & =A(\varrho) x_{k}+f\left(x_{k}, u_{k}\right) \\
y_{k} & =H x_{k}
\end{aligned}
$$

where $E=\left[\begin{array}{ll}I_{n} & -B\end{array}\right], A(\varrho)=\left[\begin{array}{ll}J(\varrho) & 0_{n \times m}\end{array}\right], H=\left[\begin{array}{ll}C & 0_{p \times m}\end{array}\right]$ and $f=\phi\left(\xi_{k}, u_{k}\right)$. The initial conditions for the system and the observer are

$$
\begin{aligned}
x_{0} & =\left[\begin{array}{llllllll}
51 & 5 & 5 & 1 & 1 & 1.4814 & 2.1956
\end{array}\right]^{T} \\
\hat{x}_{0} & =\left[\begin{array}{lllllll}
100 & 10 & 10 & 5 & 5 & 5 & 5
\end{array}\right]^{T}
\end{aligned}
$$

with $T_{s}=0.004 s$. The Lipschitz constant of $f$ is $\gamma=0.186$. Note that matrix $\left[\begin{array}{c}E \\ H\end{array}\right]$ is of full column rank, then the condition (3) is verified. From (7), we can deduce that

$$
T=\left[\begin{array}{ccccc}
1 & 0 & 0 & 0 & 0 \\
0 & 0 & 0 & 0 & 0 \\
0 & 0 & 0 & 0 & 0 \\
0 & 0 & 0 & 1 & 0 \\
0 & 0 & 0 & 0 & 0.5 \\
0 & -1 & 0 & 0 & 0 \\
0 & 0 & -1 & 0 & 0
\end{array}\right], \quad Q=\left[\begin{array}{ccc}
0 & 0 & 0 \\
1 & 0 & 0 \\
0 & 1 & 0 \\
0 & 0 & 0 \\
0 & 0 & 0.5 \\
1 & 0 & 0 \\
0 & 1 & 0
\end{array}\right] .
$$

We consider that available measurements are perturbed by a Gaussian white noise whose empirical standard deviations is $10 \%$ of that of $y$. We obtain, after solving the LMIs 19 ) and (20) using YALMIP, a toolbox for modeling and optimization in Matlab/Simulink (http://control.ee.ethz.ch/ joloef/wiki/pmwiki.php), the following results

$$
\begin{aligned}
P & =\left[\begin{array}{ccccccc}
1.38 & 0 & 0 & 0 & 0 & 0 & 0 \\
0 & 3.52 & 0 & 0 & 0 & 0 & 0 \\
0 & 0 & 3.52 & 0 & 0 & 0 & 0 \\
0 & 0 & 0 & 3.23 & 0 & 0 & 0.32 \\
0 & 0 & 0 & 0 & 3.67 & 0 & 0 \\
0 & 0 & 0 & 0 & 0 & 3.214 & 0 \\
0 & 0 & 0 & 0.32 & 0 & 0 & 2.4
\end{array}\right], \\
R_{0} & =\left[\begin{array}{ccccccc}
0 & 0 & 0 & 0 & 0 & -3.21 & 0 \\
0 & 0 & 0 & -0.32 & 0 & 0 & -2.4 \\
0 & 0 & 0 & 0 & 0.07 & 0 & 0
\end{array}\right], R_{1}=10^{-3}\left[\begin{array}{ccccccc}
0 & 0 & 0 & 0 & 0 & 12.9 & 0 \\
0 & 0 & 0 & 1.3 & 0 & 0 & 9.6 \\
0 & 0 & 0 & 0 & -7.3 & 0 & 0
\end{array}\right],
\end{aligned}
$$




$$
\begin{gathered}
F_{0}=\left[\begin{array}{ccc}
0 & 0 & 0 \\
0 & 0 & 0 \\
0 & 0 & 0 \\
0 & 0 & 0 \\
0 & 0 & 0.02 \\
-1 & 0 & 0 \\
0 & -1 & 0
\end{array}\right], F_{1}=10^{-3}\left[\begin{array}{ccc}
0 & 0 & 0 \\
0 & 0 & 0 \\
0 & 0 & 0 \\
0 & 0 & 0 \\
0 & 0 & -2 \\
4 & 0 & 0 \\
0 & 4 & 0
\end{array}\right], L_{0}=\left[\begin{array}{ccc}
0 & 0 & 0 \\
0 & 0 & 0 \\
0 & 0 & 0 \\
0 & 0 & 0 \\
0 & 0 & 0.02 \\
-1 & 0 & 0 \\
0 & -1 & 0
\end{array}\right], \\
L_{1}=10^{-3}\left[\begin{array}{ccc}
0 & 0 & 0 \\
0 & 0 & 0 \\
0 & 0 & 0 \\
0 & 0 & 0 \\
0 & 0 & -2 \\
4 & 0 & 0 \\
0 & 4 & 0
\end{array}\right] \text { and } \tau=5.9473 .
\end{gathered}
$$

Considering a "dry weather" period, the simulation results based on the obtained observer are presented in figures 2 8 . The results of estimation of state variables $X_{D C O}$, $S_{N O}, S_{N H}, S_{N D}$ and $S_{O}$ are presented in figures 2 6 . The unknown inputs $S_{N O}^{i n}$ and $S_{N H}^{i n}$ and their estimates are given by figures 7 8 . Figure 9 shows the estimation errors. These figures show the performances of the presented estimation method.

\section{Conclusion}

In this paper, a nonlinear observer for Lipschitz discrete-time systems using LPV approach is presented with application to a nonlinear reduced model of an activated sludge process. The stability analysis is performed using the Lyapunov function that leads to the linear matrix inequalities (LMIs) formulation to get the observer. Performances of the proposed observer have been shown through the simulation results. These figures show the performances of the presented estimation method.

\section{Acknowledgments}

This work is supported by the Ministry for the Culture, the Higher Education and Research of Luxembourg (BFR 04/117) and FNR (Fonds National de la Recherche) of Luxembourg.

\section{References}

[1] A. Alessandri Design of observers for Lipschitz nonlinear systems using LMI. In NOLCOS, IFAC Symposium on Nonlinear Control Systems, pages 603-608, Stuttgart, Germany, 2004.

[2] A. Zemouche and M. Boutayeb. Observer design for Lipschitz nonlinear systems. the discrete-time case. IEEE Transactions on Circuits and Systems II Analog and Digital Signal Processing, 53(8):777-781, 2006.

[3] J. Alex, J.F. Beteau, J.B. Copp, C. Hellinga, U. Jeppsson, S. Marsili-Libelli, M.N. Pons, H. Spanjers, and H. Vanhooren. Benchmark for evaluating control strategies in wastewater treatment plants. In European Control Conference 1999 , ECC'99, Karlsruhe, Germany, August 31- September 31999.

[4] M. Abbaszadeh and H.J. Marquez. Robust $H_{\infty}$ observer design for sampled-data Lipschitz nonlinear systems with exact and Euler approximate models. Automatica, 44:799-806, 2008. 
[5] G.I. Bara, J. Daafouz, and F. Kratz. Advanced gain scheduling techniques for the design of parameter-dependent observers. In Proceedings of the 40th IEEE Conference on Decision and Control, Orlando,USA, Decembre 04 - 072001.

[6] B. Boulkroune, M. Darouach, M. Zasadzinski, and S. Gillé. State and unknown input estimation for nonlinear singular systems: application to the reduced model of the activated sludge process. In 16th IEEE Mediterranean Conference on Control and Automation, Ajaccio, France, June 25-27 2008.

[7] S.P. Boyd, L. El Ghaoui, E. Féron, and V. Balakrishnan. Linear Matrix Inequality in Systems and Control Theory. SIAM, Philadelphia, 1994.

[8] C. Califano, S. Monaco, and D. Normand-Cyrot. On the observer design in discretetime. Syst. \& Contr. Letters, 49(4):255-265, 2001.

[9] B. Chachuat. Méthodologie d'optimisation dynamique et de commande optimale des petites stations d'épuration à boues activées. $\mathrm{PhD}$ thesis, Institut National Polytechnique de Lorraine, France, 2001.

[10] B. Chachuat, N. Roche, and M. A. Latifi. Reduction of the asm1 model for optimal control of small-size activated sludge treatment plants. Revue des sciences de l'eau / Journal of Water Science, 16(1):05-26, 2003.

[11] D. Dochain. State and parameter estimation in chemical and biochemical processes: a tutorial. Journal of Process Control, 13(8):801-818, 2003.

[12] M. Darouach and M. Boutayeb. Design of observers for descriptor systems. IEEE Trans. Aut. Contr., 40:1323-1327, 1995.

[13] M. Darouach, M. Zasadzinski and D. Mehdi. State estimation of stochastic singular linear systems. Int. J. Syst. Sci., 24(2):345-354, 1993.

[14] C. Gómez-Quintero and I. Queinnec. State and disturbance estimation for an alternating activated sludge process. In Proc. of the 15th triennal World IFAC Congress, Barcelone, Espagne, 21-26 juillet 2001.

[15] C. Gómez-Quintero, I. Queinnec, and J. P. Babary. A reduced nonlinear model of an activated sludge process. In Int. Symp. on Advanced Control on Chemical Processes, pages 1037-1042, Pisa, Italy, 2000.

[16] W. Gujer, M. Henze, T. Mino, and M. C. M. Van Loosdrecht. Activated sludge model no.3. Water Science and Technology, 39:183-193, 1999.

[17] M. Henze, C. P. L. Grady, W. Jr., Gujer, G. V. R. Marais, and T. Matsuo. Activated sludge model No.1. IAWQ Scientific and Technical Report No.1, London, UK, 1987.

[18] M. Henze, W. Gujer, T. Mino, T. Matsuo, M. C. M. Wentzel, and G. V. R. Marais. Activated sludge model No.2. IAWQ Scientific and Technical Report No.3, London, UK, 1995.

[19] M. Henze, W. Gujer, T. Mino, T. Matsuo, M. C. Wetzel, G. V. R. Marais, and M. C. M. van Loosdrecht. Activated sludge process model No. 2d. Water Science and Technology, 39(1):165-182, 1999. 
[20] S. Ibrir. Circle-criterion approach to discrete-time nonlinear observer design. Automatica, 43(8):1432-1441, 2007.

[21] U. Jeppsson and G. Olsson. Reduced order models for online parameter identification of the actived sludge process. Water Science and Technology, 28:173-183, 1993.

[22] N. Kazantzis and C. Kravaris. Discrete-time nonlinear observer design using functional equations. Syst. \& Contr. Letters, 42(2):81-94, 2001.

[23] L.J.S. Lukasse, K.J. Keesman, and G. Van Straten. A recursively identified model for short term predictions of $\mathrm{Nh}_{4} / \mathrm{No}_{3}$ concentrations in alternating activated sludge processes. Journal of Process Control, 9(1):87-100, 1999.

[24] M. Boutayeb, M. Darouach, and H. Rafaralahy. Generalized state-space observers for chaotic synchronization and secure communication. IEEE Trans. Circ. Syst. I : Fund. Theory \& Appli., 49:345-349, 2002.

[25] M. Mulas, S. Tronci, and R. Baratti. Development of a 4-measurable states activated sludge process model deduced from the ASM1. In 8th International Symposium on Dynamics and Control of Process Systems, pages 213-218, Cancun, Mexico, 2007.

[26] R. Prabhakar, R. Pagilla, and Y. Zhu. Controller and observer design for Lipschitz nonlinear systems. In IEEE American Control Conference, pages 2379-2384, Boston, Massachusetts, 2004.

[27] R. Rajamani. Observer for Lipschitz nonlinear systems. IEEE Trans. Aut. Contr., 43:397-401, 1998.

[28] K. Reif, F. Sonnemann, and R. Unbehauen. Nonlinear State Observation Using $H_{\infty^{-}}$ Filtering Riccati Design. IEEE Transactions on Automatic Control, 44(1):203-208, 1999.

[29] S. Xu, J. Lu, S. Zhou, and C. Yang. Design of observers for a class of discretetime uncertain nonlinear systems with time delay. Journal of the Franklin Institute, 341(3):295-308, 2004.

[30] I. Y. Smets, J. V. Haegebaert, R. Carrette, and J. F. Van Impe. Linearization of the activated sludge model ASM1 for fast and reliable predictions. Water Res, 37(8):18311851, 2003.

[31] M. A. Steffens, P. A. Lant, and R. B. Newell. A systematic approach for reducing complex biological wastewater treatment model. Water Science and Technology, 31(3):590-606, 1997.

[32] H. Zhao and M. Kümmel. State and parameter estimation for phosphorus removal in an alternating activated sludge process. J. Proc. Control, 5(5):341-351, 1995.

[33] F. Zhu and Z. Han. A note on observers for Lipschitz nonlinear systems. IEEE Trans. Aut. Contr., 47:1751-1754, 2002.

[34] R. Chiu, J.L. Navarro and J. Pico. A nonlinear observer for bioprocesses using LMI. In 10th International IFAC Symposium on Computer Applications in Biotechnology, pages 389-394, Cancun, Mexico, 2007. 


\section{Nomenclature}

$S_{I} \quad$ Concentration of soluble inert organic matter

$\left[m g / l^{-1}\right]$

$S_{S} \quad$ Concentration of readily biodegradable substrate

$\left[m g / l^{-1}\right]$

$S_{O}$

Concentration of dissolved oxygen

$\left[m g / l^{-1}\right]$

$S_{O}^{s a t}$

Dissolved oxygen saturation concentration

$\left[m g / l^{-1}\right]$

$S_{N O}$

Concentration of nitrate and nitrite nitrogen

$\left[m g / l^{-1}\right]$

Concentration of ammonia nitrogen

$\left[m g / l^{-1}\right]$

$S_{N H}$

Concentration of soluble biodegradable organic nitrogen

$\left[\mathrm{mg} / \mathrm{l}^{-1}\right]$

Concentration of particulate inert organic matter

$\left[m g / l^{-1}\right]$

$X_{I}$

Concentration of slowly biodegradable substrate

$\left[m g / l^{-1}\right]$

$X_{s}$

Concentration of active heterotrophic biomass

$\left[\mathrm{mg} / \mathrm{l}^{-1}\right]$

$X_{B, A}$

Concentration of active autotrophic biomass

$\left[\mathrm{mg} / \mathrm{l}^{-1}\right]$

$X_{N D}$

Concentration of particulate biodegradable organic nitrogen

$b_{A}$

Decay rate coefficient for autotrophic organisms

$b_{H}$

$f_{r X I}$

Decay rate coefficient for heterotrophic organisms

Fraction of biomass generating the particulate products

$\left[m g / l^{-1}\right]$

$\left[d^{-1}\right]$

$\left[d^{-1}\right]$

$[-]$

$i_{N B M}$

Mass of nitrogen in the biomass

$i_{N X I}$

$k_{L^{a}}$

Mass of nitrogen in the inert particulate organic matter

$\left[g_{N} g_{C O D}^{-1}\right]$

$\left[g_{N} g_{C O D}^{-1}\right]$

Coefficient of oxygen rate

$K_{(.)}$

$K_{N H, A}$

Half-saturation coefficient :

$K_{N 0}$

of ammonia for autotrophs

$K_{O, A}$

of nitrate for denitrifying heterotrophs

$K_{O, H}$

of oxygen for autotrophs

$K_{S}$

of oxygen for heterotrophs

$K_{X}$

for heterotrophic organisms

$Y_{A}$

for hydrolysis of slowly biodegradable substrate

$Y_{H}$

Yield coefficient for autotrophic organisms

Yield coefficient for heterotrophic organisms

$\mu_{A}$

$\mu_{H}$

Maximum specific growth rate for autotrophic organisms

$\eta_{N O_{3} g}$

$\eta_{N_{3}} h$

$V_{O}$

Maximum specific growth rate for heterotrophic organisms

$\left[d^{-1}\right]$

$\left[g_{N H} m^{-3}\right]$
$\left[g_{N 0} m^{-3}\right]$
$\left[g_{O_{2}} m^{-3}\right]$
$\left[g_{O_{2}} m^{-3}\right]$
$\left[g_{D C O} m^{-3}\right]$
$\left[g_{D C O} g_{D C O}^{-1}\right]$
$[-]$
$[-]$
$\left[d^{-1}\right]$
$\left[d^{-1}\right]$
$[-]$
$[-]$
$\left[m^{3}\right]$
$\left[m^{3} d^{-1}\right]$ 


\begin{tabular}{|c|c|c|}
\hline Parameter & Value & Range of variation \\
\hline$Y_{H}$ & 0.67 & $0.38-0.75$ \\
\hline$i_{N B M}$ & 0.08 & - \\
\hline$K_{S}$ & 10 & $5-225$ \\
\hline$K_{O, H}$ & 0.20 & $0.01-0.20$ \\
\hline$K_{N O}$ & 0.50 & $0.01-0.50$ \\
\hline$K_{N H, A}$ & 1.0 & - \\
\hline$K_{O, A}$ & 0.40 & $0.40-2.0$ \\
\hline$\eta_{N O, g}$ & 0.8 & $0.6-1.0$ \\
\hline$\eta_{N O, h}$ & 0.8 & - \\
\hline$Y_{A}$ & 0.24 & $0.07-0.28$ \\
\hline$f r_{X I}$ & 0.08 & - \\
\hline$\mu_{H}$ & 4.0 & $0.60-13.2$ \\
\hline$b_{H}$ & 0.30 & $0.05-1.6$ \\
\hline$\mu_{A}$ & 0.5 & $0.20-1.0$ \\
\hline$b_{A}$ & 0.05 & $0.05-0.3$ \\
\hline$\kappa_{a}$ & 0.05 & - \\
\hline$\kappa_{h}$ & 3.0 & - \\
\hline$f_{S S}$ & 0.79 & - \\
\hline
\end{tabular}

\begin{tabular}{|c|c|}
\hline Parameter & Value \\
\hline$\theta_{1}$ & 9956 \\
\hline$\theta_{2}$ & 693 \\
\hline$\theta_{3}$ & 283 \\
\hline$\theta_{4}$ & 124 \\
\hline$\theta_{5}$ & 480 \\
\hline$K_{D C O}$ & 220 \\
\hline$K_{N D}$ & 258 \\
\hline$X_{B, A}$ & $136 g_{D C O} \cdot m^{-3}$ \\
\hline$X_{B, H}$ & $2489 g_{D C O} \cdot m^{-3}$ \\
\hline$X_{N D}$ & $6 g_{N} \cdot m^{-3}$ \\
\hline$k_{L^{a}}$ & $240 d^{-1}$ \\
\hline$V_{O}$ & $1333 m^{3}$ \\
\hline
\end{tabular}

Table 2: Different parameters values

Table 1: Kinetic and stoechiometric parameters of the ASM1 model

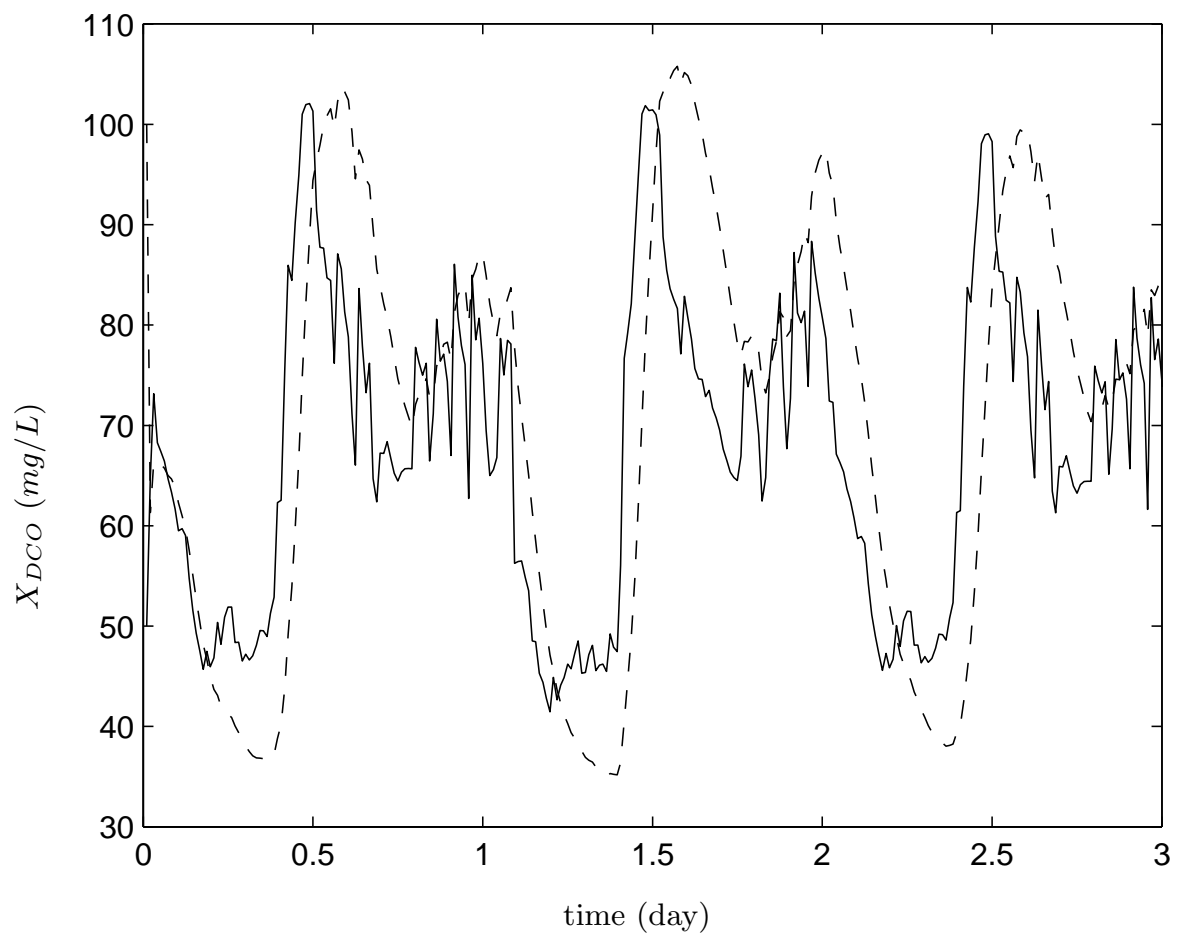

Figure 2: The concentration of $X_{D C O}$ (solid line) and its estimate (dashed line) 


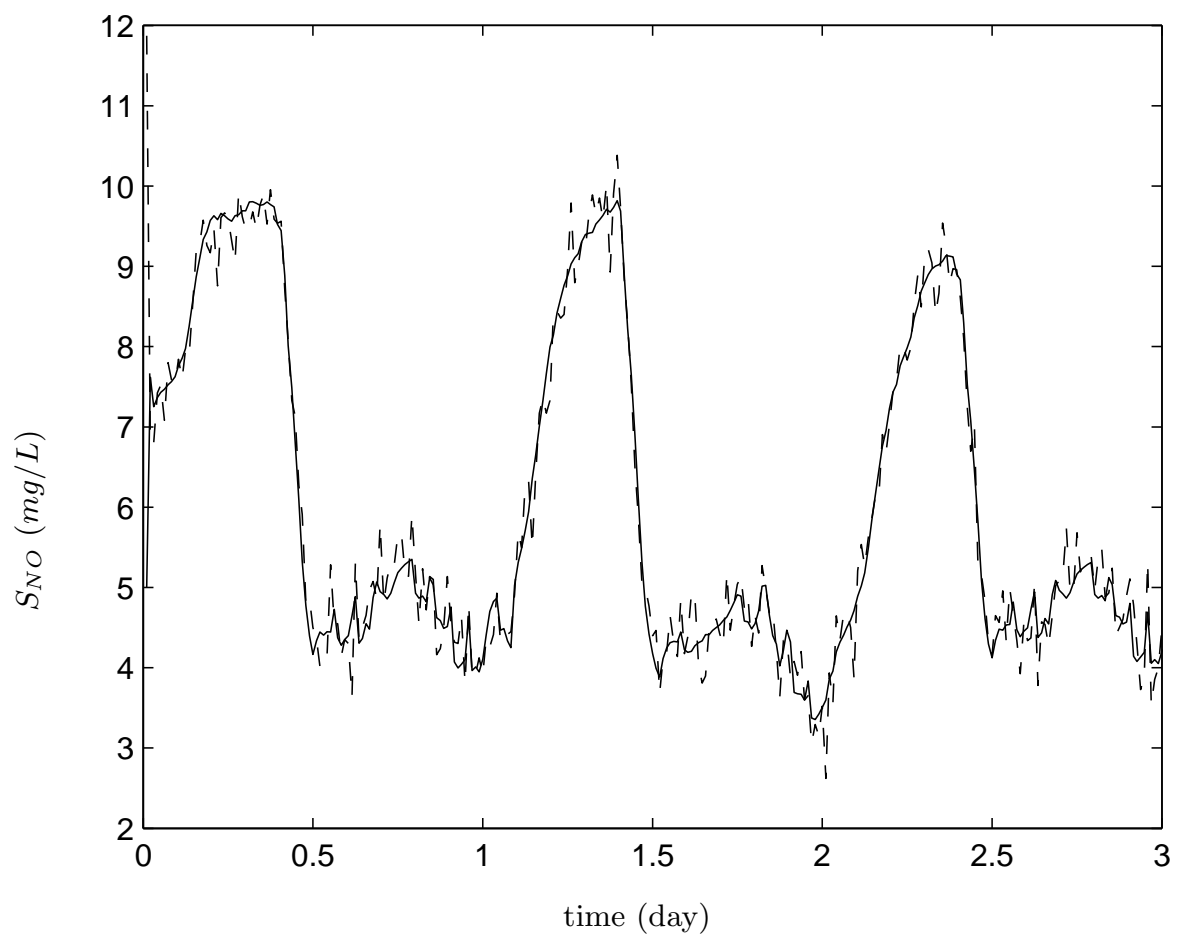

Figure 3: The concentration of $S_{N O}$ (solid line) and its estimate (dashed line)

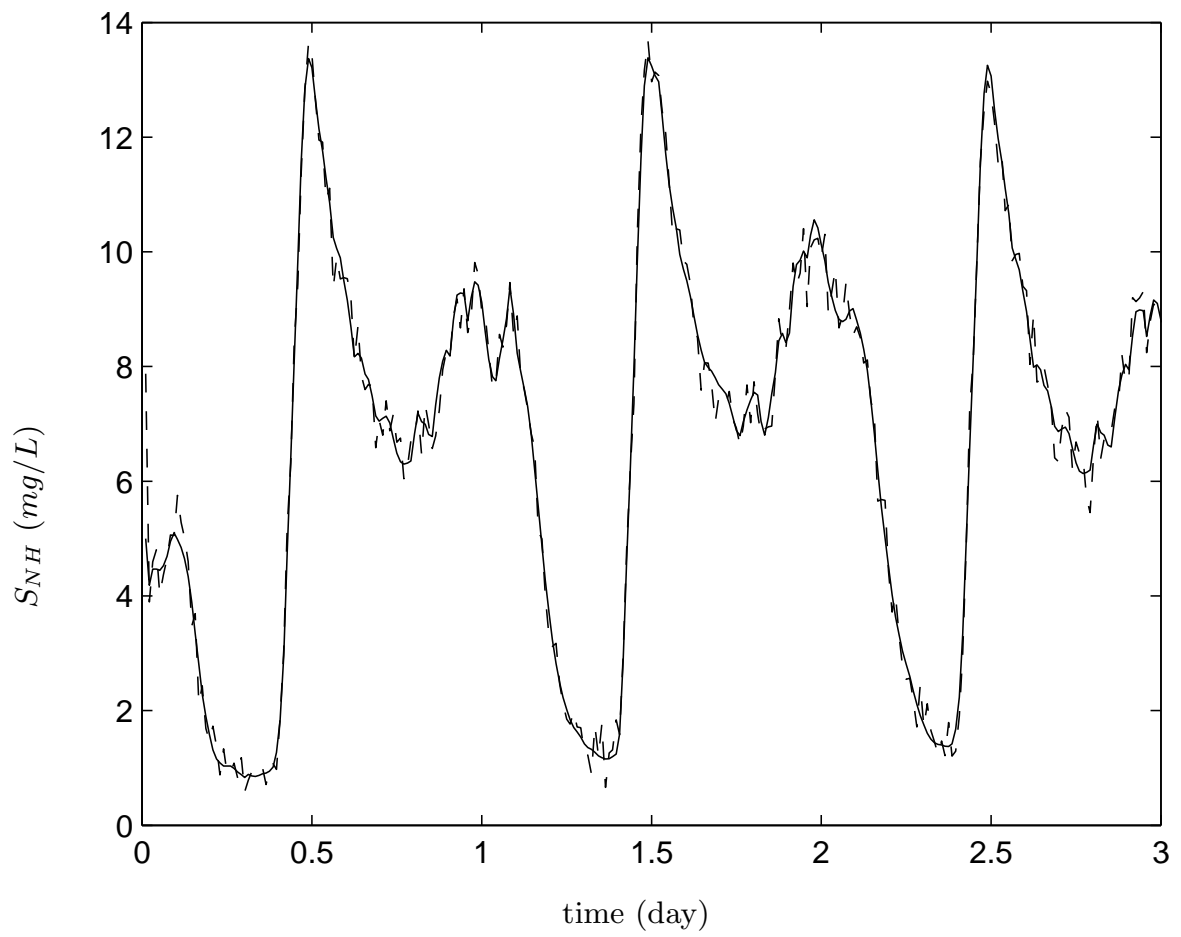

Figure 4: The concentration of $S_{N H}$ (solid line) and its estimate (dashed line) 


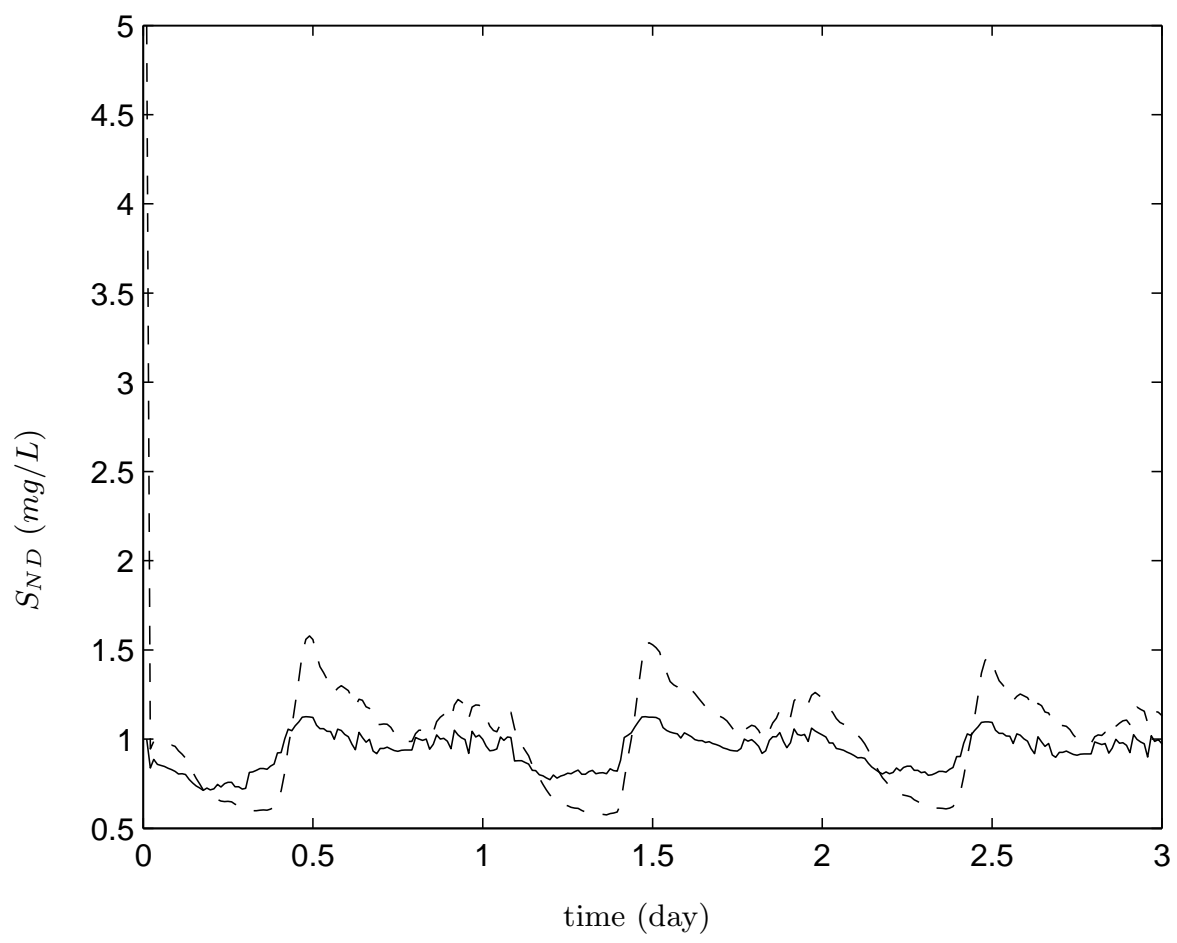

Figure 5: The concentration of $S_{N D}$ (solid line) and its estimate (dashed line)

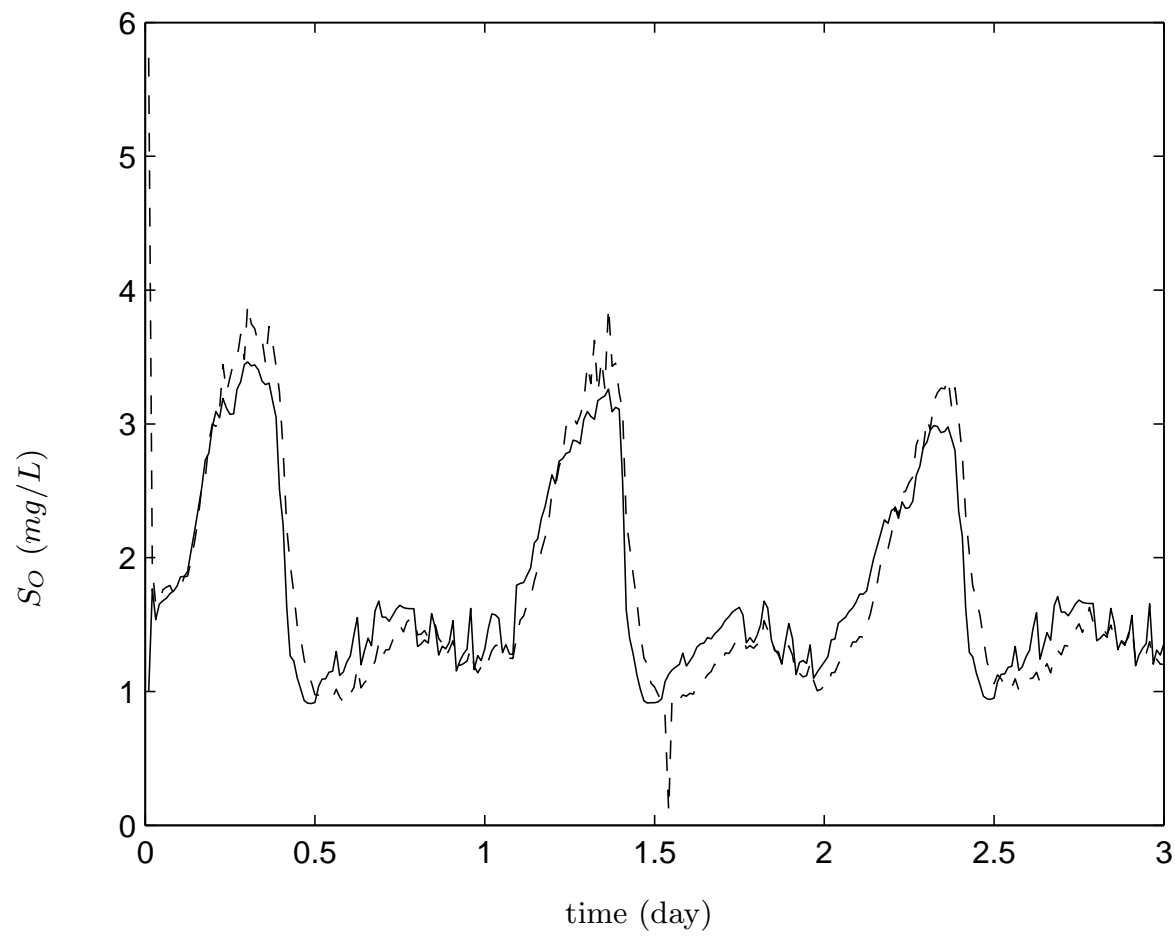

Figure 6: The concentration of $S_{O}$ (solid line) and its estimate (dashed line) 


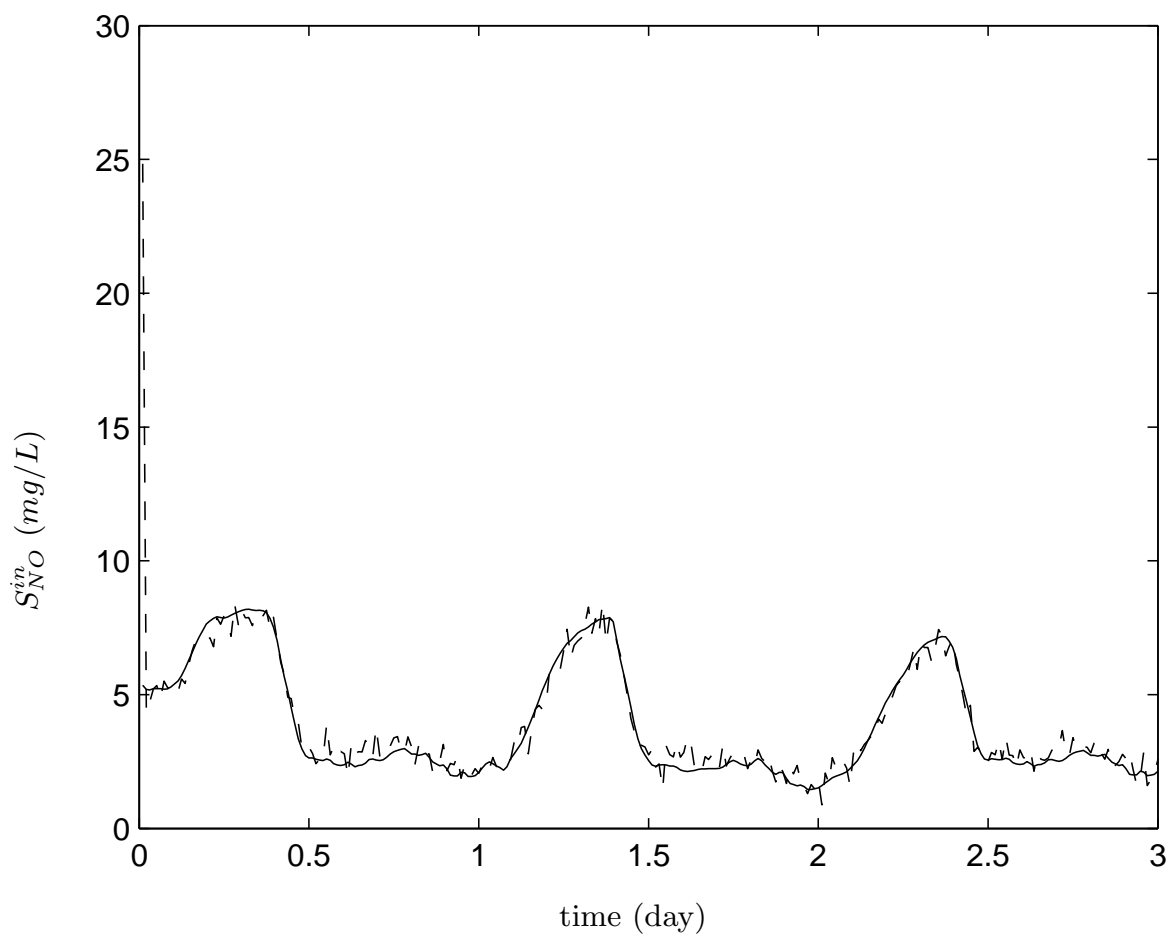

Figure 7: Unknown input $S_{N O}^{i n}$ (solid line) and its estimate (dashed line)

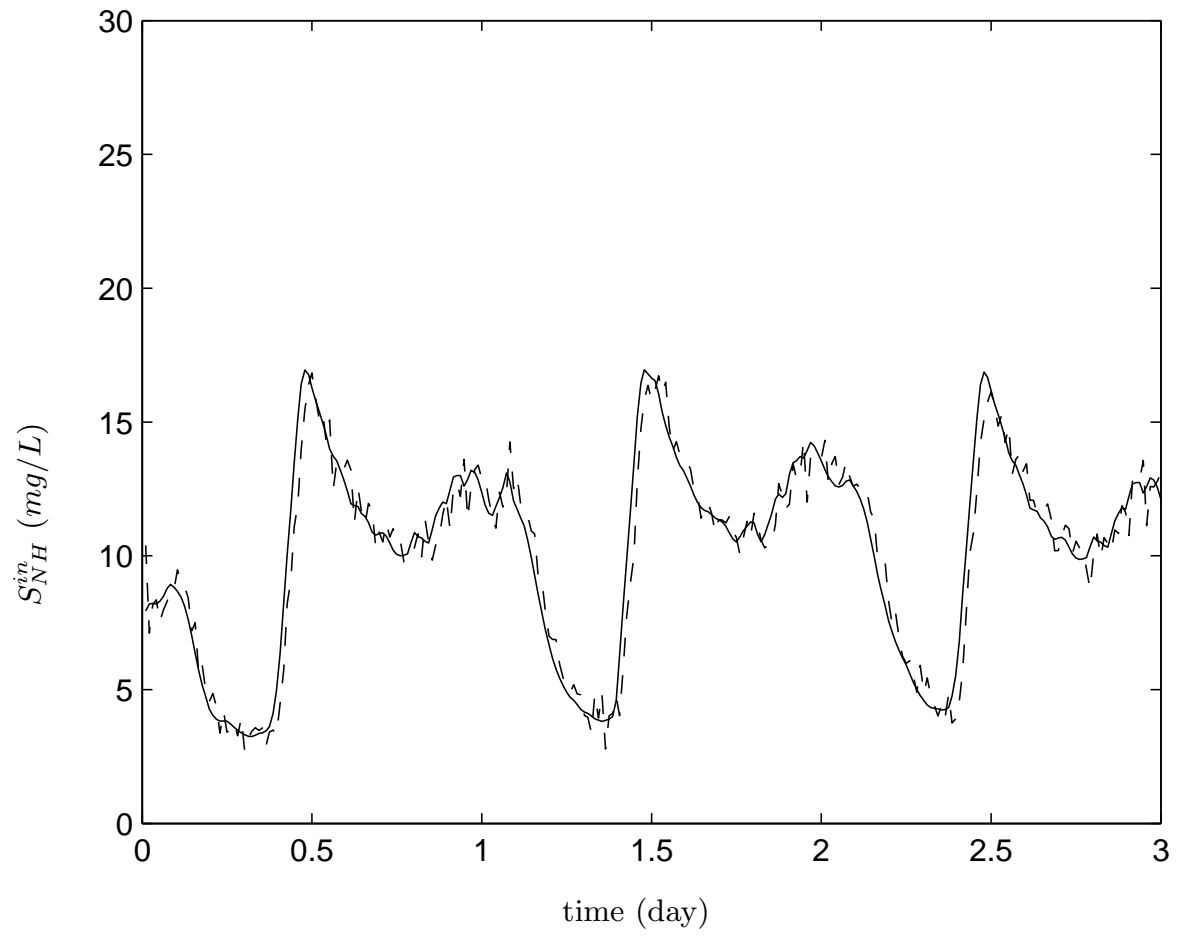

Figure 8: Unknown input $S_{N H}^{i n}$ (solid line) and its estimate (dashed line) 


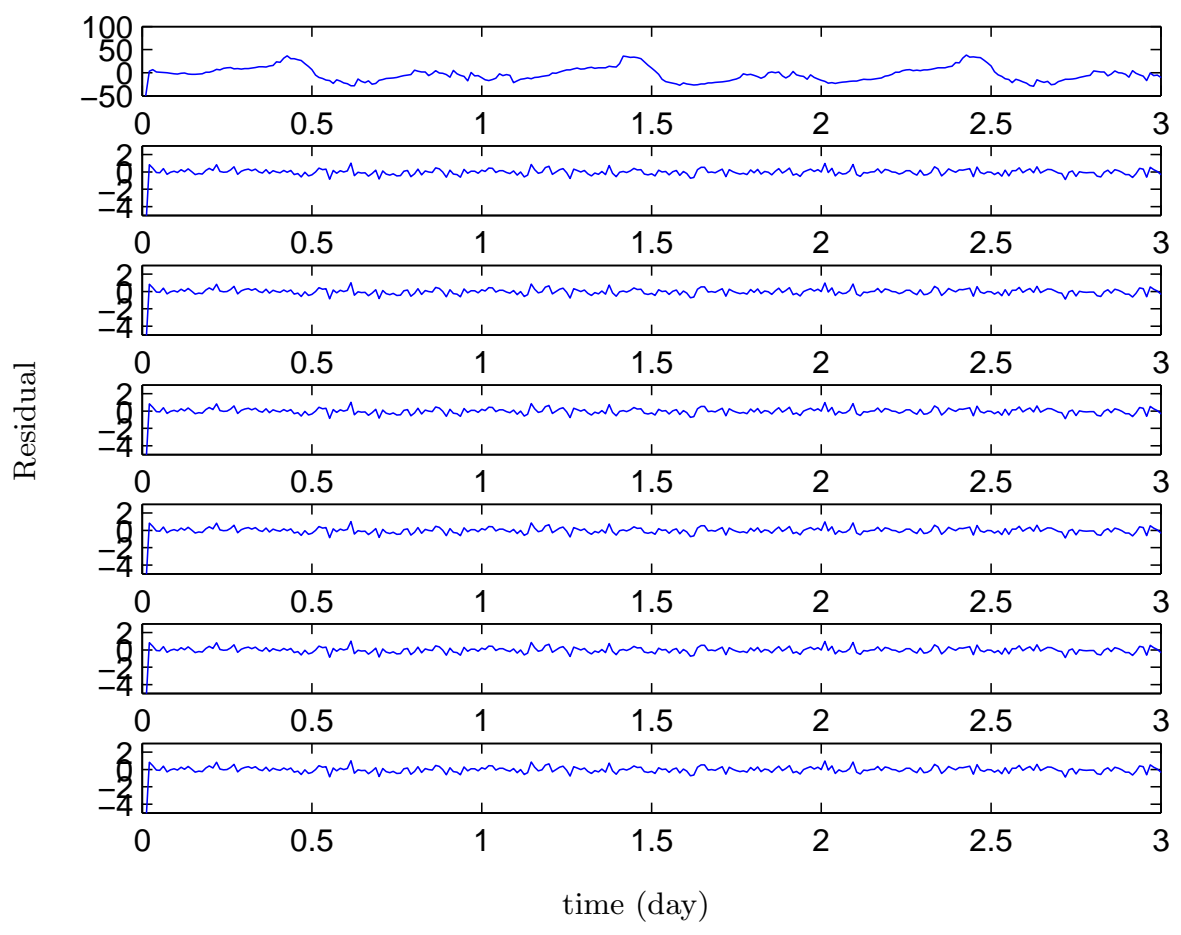

Figure 9: Estimation errors 